\title{
Sparse Feature Selection Identifies H2A.Z as a Novel Pattern-Specific Biomarker for Asymmetrically Self-Renewing Distributed Stem Cells
}

\author{
Yang Hoon Huh ${ }^{1}$, Minsoo Noh ${ }^{2}$, Frank R. Burden ${ }^{3}$, Jennifer C. Chen ${ }^{4}$, David A. Winkler ${ }^{3,5,6, *}$, and \\ James L. Sherley ${ }^{7, *}$, Hee-Seok Kweon ${ }^{1, *}$ \\ 1. Division of Electron Microscopic Research, Korea Basic Science Institute, 169-148 Gwahak-ro, \\ Yuseong-gu, Daejeon, Korea \\ 2. College of Pharmacy, Seoul National University, Seoul, Republic of Korea \\ 3. CSIRO Manufacturing Flagship, Clayton, Australia \\ 4. The Senator Paul D. Wellstone Muscular Dystrophy Cooperative Research Center, University of \\ Massachusetts Medical School, Worcester, MA, USA \\ 5. Monash Institute of Pharmaceutical Sciences, Parkville, Australia \\ ${ }^{6 .}$ Latrobe Institute for Molecular Science, Bundoora, Australia \\ 7. Asymmetrex, LLC Boston, MA, USA
}

Despite the important role of distributed stem cell (DSCs) in homeostatic tissue cell renewal and tissue repair, and their use in currently available cell replacement therapies (e.g., hematopoietic stem cell transplantation), there are no effective means for accurate determination of their numbers for medical applications. Therefore, there is a long-standing unmet clinical need for biomarkers with high specificity for DSCs in tissues, or for use in diagnostic and therapeutic cell preparations (e.g., bone marrow). Previous searches for these illusive biomarkers relied on global gene expression profiles for DSCs that were based on comparisons of genes expressed in embryonic stem cells (ESCs) and genes expressed in cell populations enriched for adult stem cells (ASCs) [1, 2, 3], a sub-category of DSCs [4, 5]. These ASC-enriched populations also contained a significant fraction of non-stem committed progenitors and differentiating progeny cells that limited their utility for identifying genes whose expression was unique to DSCs, i.e., "stemness genes" [1, 2, 3]. To identify DSC such useful and specific biomarkers, we combined a novel sparse feature selection method with combinatorial molecular expression data focused on asymmetric self-renewal, is a conspicuous property of DSCs. The analysis identified reduced expression of the histone H2A variant H2A.Z as a superior molecular discriminator for DSC asymmetric self-renewal. Subsequent molecular expression studies showed H2A.Z to be a novel "pattern-specific biomarker" for asymmetrically self-renewing cells with sufficient specificity to count asymmetrically self-renewing DSCs in vitro and potentially in situ [6].

\section{References:}

[1] NB Ivanova et al., Science 298 (2002), p. 601-604.

[2] M Ramalho-Santos et al., Science 298 (2002), p. 597-600.

[3] NO Fortunel et al., Science 302 (2003), p. 393.

[4] JL Sherley, Breast Dis. 29 (2008), p. 37-46.

[5] M Noh et al., Plos ONE 6 (2011), e22077.

[6] This work was supported by NIH-NHGRI grant \#PSO HG 003170, NIH-NIEHS grant \#689471, and NIH-NIGMS Director's Pioneer Award \#5DP1OD000805. JCC was supported by the Eunice Kennedy Shriver National Institute of Child Health \& Human Development of the NIH (\#U54HD060848). DAW also acknowledges support from the CSIRO Newton Turner Award for Exceptional Senior Scientists. HSK was supported by the KBSI grant \#35700. 


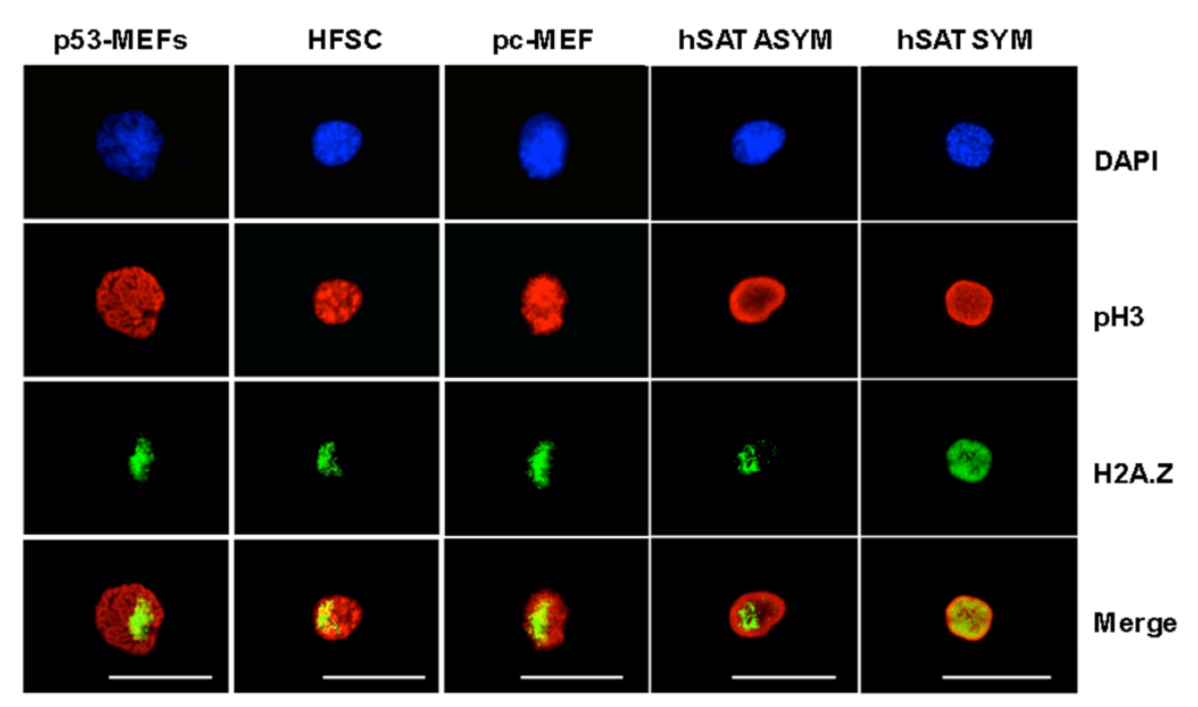

Figure 1. H2A.Z asymmetry is detected in prophase nuclei, identified by phosphorylated histone H3, for a variety of cultured cell types. p53-MEFs, example of H2A.Z asymmetry in genetically engineered mouse embryo fibroblasts (line Ind-8) under conditions that promote asymmetric self-renewal. HFSC, example of H2A.Z asymmetry in mouse hair follicle stem cells (strain 3C5) under conditions that promote asymmetric self-renewal. pc-MEF, example of cell with H2A.Z asymmetry detected in cultures of pre-crises MEFs. In cultures enriched for human skeletal muscle satellite stem cells: hSAT ASYM, example of cell with H2A.Z asymmetry, and hSAT SYM, example of cell with symmetric H2A.Z. Scale bar $=25$ microns.
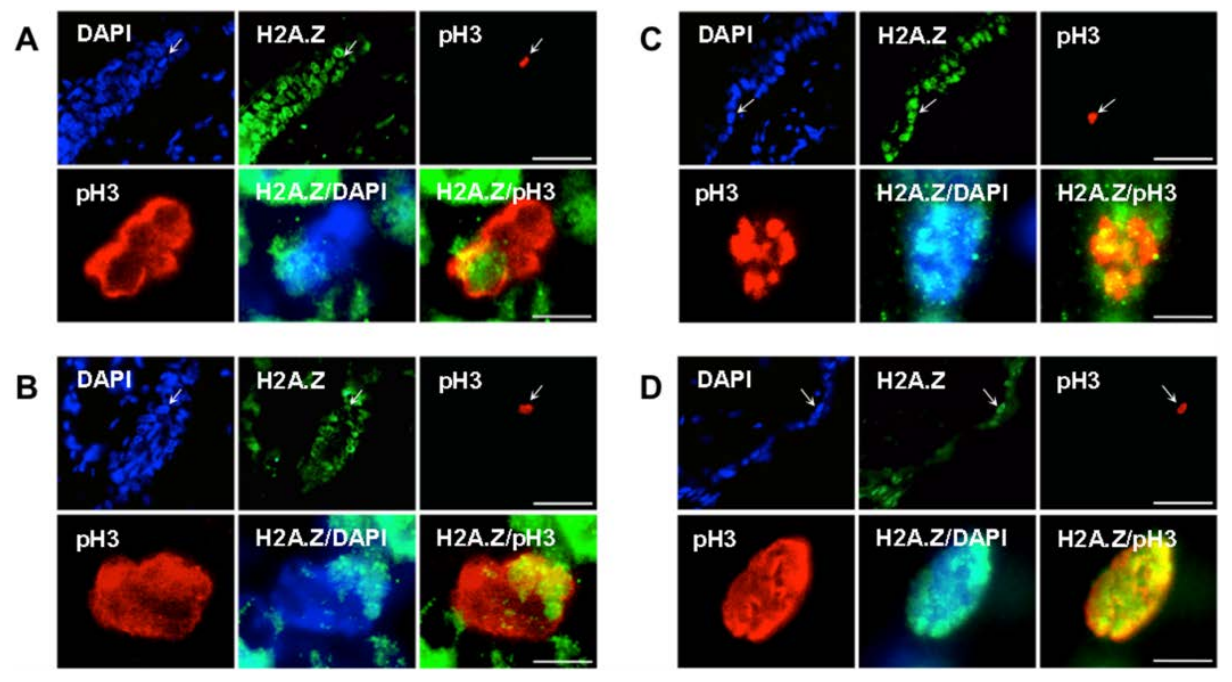

Figure 2. Detection of mitotic cells with H2A.Z asymmetry in mouse hair follicles. Ten-micron thick, paraffin-embedded sections of adult mouse skin were evaluated by ISIF with antibodies specific for H2A.Z and phosphorylated histone H3 (pH3), a biomarker for mitotic cells. A and B, examples of mitotic hair follicle cells with H2A.Z asymmetry. C and D, examples of epidermal mitotic cells with symmetric H2A.Z. Top rows, low magnification images; scale bars $=50$ microns. Bottom rows, $10 \mathrm{X}$ magnification images of the cell identified with arrows in upper images; scale bars $=5$ microns. 